\title{
RESEARCH ON HORIZONTAL ACCURACY METHOD OF HIGH SPATIAL RESOLUTION REMOTELY SENSED ORTHOPHOTO IMAGE
}

\author{
Y. M. Xu ${ }^{1}$, J.X.Zhang ${ }^{1}$, F. Yu ${ }^{2}$, S. Dong ${ }^{1}$ \\ ${ }^{1}$ National Quality inspection and Testing Center for Surveying and Mapping Products, (xuym, zhangjx, dongs)@ sbsm.gov.cn \\ ${ }^{2}$ Chinese Academy of Surveying and Mapping, yufan021@126.com
}

Commission III, WG II/IVb

KEY WORDS: Horizontal Accuracy, Orthophoto Image, Qualitty Inspection, Testing Point

\begin{abstract}
:
At present, in the inspection and acceptance of high spatial resolution remotly sensed orthophoto image, the horizontal accuracy detection is testing and evaluating the accuracy of images, which mostly based on a set of testing points with the same accuracy and reliability. However, it is difficult to get a set of testing points with the same accuracy and reliability in the areas where the field measurement is difficult and the reference data with high accuracy is not enough. So it is difficult to test and evaluate the horizontal accuracy of the orthophoto image. The uncertainty of the horizontal accuracy has become a bottleneck for the application of satellite borne high-resolution remote sensing image and the scope of service expansion. Therefore, this paper proposes a new method to test the horizontal accuracy of orthophoto image. This method using the testing points with different accuracy and reliability. These points' source is high accuracy reference data and field measurement. The new method solves the horizontal accuracy detection of the orthophoto image in the difficult areas and provides the basis for providing reliable orthophoto images to the users.
\end{abstract}

\section{INTRODUCTION}

Full papers submitted for double-blind review to the Annals must not contain any information which makes it possible to identify the authors. In particular, names and affiliations must be removed from the manuscript submitted for review. Also sentences such as "As we have shown in previous work (Author_x, 20xx)" are to be avoided. Instead use a formulation such as "Author_x (20xx) has shown ...". Note that submissions which have not been appropriately anonymised may be subject to immediate rejection. With the development of satellite remote sensing technology, remotely sensed image has become an important data source for human to get spatial information. The applications of high spatial resolution remotely sensed orthophotos image made by spaceborne remote sensing image are becoming more and more widespread, For example, it was widely used in the fields of land and surveying and mapping. In recent years, high spatial resolution remotely sensed orthophoto image has become the main data source of the national census geography, the geographical conditions monitoring, and the national land survey, and so on. With the deep application of high spatial resolution remotely sensed orthophoto image, it's quality has attracted more attention. As a key indicator of orthophoto image's quality, the horizontal accuracy detection is essential for every image quality assessment. The traditional accuracy detection requires a set of testing points with the same accuracy and reliability, and the number and the distribution of the testing points are required. However, it is very difficult to find a set of testing points to meet the requirements in areas with relatively backward economy and hard natural conditions, and the horizontal accuracy cannot evaluated.

1
The uncertainty of horizontal accuracy has become a bottleneck for the application of satellite borne high-resolution remotely sensed image and the scope of service expansion.Therefore, this paper proposes a new method to test the horizontal accuracy of orthophoto image based on high accuracy reference data and few field measurement points which have different accuracy and reliability. To some extent, this method solves the evaluation of the horizontal accuracy of the orthophoto image in the difficult areas and provides the basis for providing reliable orthophoto images to the users.

\section{CHARACTERISTICS OF HIGH SPATIAL RESOLUTION REMOTELY SENSED ORTHOPHOTO IMAGE}

Now, high spatial resolution remotely sensed orthophoto Images are mostly based on satellite remotely sensed images, which are processed by orthophoto correction, image enhancement, image fusion and cutting. Their storage units are mostly image with standard 1:50000 segmentation, landscape and county etc. The process, method and data processing algorithm of making high spatial resolution remotely sensed orthophoto images from satellite sensing images are different from the traditional aerial images. Especially in geometric correction, It makes full use of track and sensor parameters and reduces the dependence on control points. Generally, single scene images usually use image data such as orbital /RPC parameters, DEM data and high accuracy image control points to correct the image. Based on multi source control data, multi scene images are set up by free network and regional network adjustment, and then ortho rectification is done. 


\section{HORIZONTAL ACCURACY METHOD OF HIGH SPATIAL RESOLUTION REMOTELY SENSED ORTHOPHOTO IMAGE}

The horizontal accuracy of remotely sensed image is also known as geometric positioning accuracy or pixel accuracy. That is to say, whether the relative position of each pixel which registered by coordinate is accurate, and whether there is an obvious aberration $^{[1]}$. The horizontal accuracy can be measured by mean square error in plane position. The mean square error in plane position is calculated by the difference between the testing point and the same point in the evaluation area.

\subsection{Testing point requirements}

Generally, the point used for plane precision testing needs to meet the requirements of the following aspects:

(1) The testing points should be evenly distributed, and the welldistributed testing point is the basic condition for measuring the quality.

(2) The number of testing points in a certain range should have statistical significance. Only the number of testing points has statistical significance, the mean error value obtained by the calculation of the difference values of these detection points is representative of the error. The number of testing points is related to the specifications of the orthophoto image, the area of image, the type of terrain, the productive mode of production, and so on. No less than 20 testing points are needed in the scope of the general horizontal accuracy assessment.

(3) The location of the testing point should be obvious. Facilitate the collection of image and reference data. To reduce identification error and gross error. The testing points are chosen at the image feature point as far as possible, such as the independent ground point, the intersecting point of linear features or the obvious ridge and valley, the obvious corner point or the inflection point of the ground objects, and so on.

\subsection{Acquisition method of testing point}

(1) The testing point can be obtained by field measurement. The testing point coordinates can be collected by the global navigation satellite system (GNSS) measurement or polar coordinate method.

(2) Comparison of existing results, we can use the high-accuracy or same-accuracy reference data to get the testing point coordinates.

\subsection{Computing method of mean square error in horizontal accuracy}

There is a coordinate component error between the testing point and the image homonym point. The component error in the $\mathrm{X}$ direction is $\Delta \mathrm{x}$, and the component error in the $\mathrm{Y}$ direction is $\triangle$ $\mathrm{y}$. So the horizontal error is $\Delta \mathrm{S}=\sqrt{\Delta \mathrm{x}^{2}+\Delta \mathrm{y}^{2}}$. Let the $\mathrm{X}$ direction coordinate value of the $\mathrm{i}$ testing point is $\mathrm{X}_{\mathrm{i}}$, and the coordinate value of the $\mathrm{Y}$ direction is $\mathrm{y}_{\mathrm{i}}$, The coordinate value of the Homonymous point on the image to be evaluated is $\mathrm{x}_{\mathrm{i}}^{\prime}, \mathrm{y}_{\mathrm{i}}^{\prime}$, Then the $\mathrm{X}$ direction difference is $\Delta \mathrm{x}_{\mathrm{i}}=\mathrm{x}_{\mathrm{i}}-\mathrm{x}_{\mathrm{i}}^{\prime}$, the $\mathrm{Y}$ direction difference is $\Delta y_{i}=y_{i}-y_{i}^{\prime}$, The horizontal error of this point is $\Delta \mathrm{S}_{\mathrm{i}}=\sqrt{\Delta \mathrm{x}_{\mathrm{i}}^{2}+\Delta \mathrm{y}_{\mathrm{i}}^{2}}$. If the number of testing points for the image to be evaluated is $\mathrm{n}$, then the mean square error in the horizontal direction of the image to be evaluated is $S=\sqrt{\frac{\sum_{i=1}^{n} \Delta S_{i}^{2}}{n}}$.

When each testing point has the same accuracy or the same reliability, the above method is used to calculate the error in the horizontal direction. However, it is difficult to get a group of testing points with the same reliability for the relatively backward economy and the areas with hard natural conditions. Therefore, this paper proposes a horizontal accuracy testing method which synthetically uses different reliability testing points, that is the comprehensive use of testing points from high precision reference data and field measurement points.

First of all, we need find the public homonym points among the field measurement, high precision reference data and the evaluating image. Calculating the difference ( $\triangle$ xir-f, $\triangle$ yirf)between the high precision reference data and the field measured point, the horizontal error $\left(\mathrm{S}_{\mathrm{ir}-\mathrm{f}}=\sqrt{\left.\Delta \mathrm{x}_{\mathrm{ir}-\mathrm{f}}^{2}+\Delta \mathrm{y}_{\mathrm{ir}-\mathrm{f}}^{2}\right)}\right.$ and the mean square error $\left(\mathrm{S}_{\mathrm{r}-\mathrm{f}}=\sqrt{\frac{\sum_{\mathrm{i}=1}^{\mathrm{n}} \mathrm{S}_{\mathrm{ir}}^{2}}{\mathrm{n}}}, \mathrm{n}\right.$ is the number of the public points) in the horizontal direction. Calculating the difference ( $\triangle$ xir, $\triangle$ yir) between the evaluating image and the high precision reference data, the horizontal error $\left(S_{\mathrm{ir}}=\right.$ $\left.\sqrt{\Delta \mathrm{x}_{\mathrm{ir}}^{2}+\Delta \mathrm{y}_{\mathrm{ir}}^{2}}\right)$ and the mean square error $\left(\mathrm{S}_{\mathrm{r}}=\sqrt{\frac{\sum_{\mathrm{i}=1}^{\mathrm{n}} \mathrm{S}_{\mathrm{ir}}^{2}}{\mathrm{n}}}, \mathrm{n}\right.$ is the number of the public points) in the horizontal direction.

Secondly, According to the mean square error value of the homonym point between high precision reference data and field point, the mean square error value of the homonym point between the evaluating image and high precision reference data, we can determine the weight of the high precision reference data testing point and the field testing point. Set the weight of the testing points from high precision reference data is $1(\operatorname{Pr}=1)$, detection point when evaluating the plane error of the image. The weight of field testing points in evaluating the horizontal error of the orthophoto image is $P_{f}=1+\frac{S_{r-f}}{s_{r-f}+S_{r}}$.

Thirdly, Calculating the difference ( $\triangle \mathrm{xf}, \triangle \mathrm{yf}$ )between the evaluating image data and the field measured point, the horizontal error $\left(\mathrm{S}_{\mathrm{f}}=\sqrt{\Delta \mathrm{x}_{\mathrm{f}}^{2}+\Delta \mathrm{y}_{\mathrm{f}}^{2}}\right)$ and the mean square error $\left(\mathrm{S}_{\mathrm{f}}=\sqrt{\frac{\sum_{\mathrm{i}=1}^{\mathrm{n}} \mathrm{S}_{\mathrm{f}}^{2}}{\mathrm{n}}}\right.$, where $\mathrm{n}$ is the number of the field measured point) in the horizontal direction.

Finally, the horizontal accuracy of high resolution remotely sensed orthophoto image is tested by using the testing points with weights.

$$
S=\sqrt{\frac{\sum_{i=1}^{n_{r}} S_{i r}^{2} \times p_{i r}+\sum_{i=1}^{n_{f}} S_{i f}^{2} \times p_{i f}}{n_{r} \times p_{r}+n_{f} \times p_{f}}}
$$

where $\mathrm{n}_{\mathrm{r}}=$ the number of testing points of large scale topographic maps ocal length

$\mathrm{n}_{\mathrm{f}}=$ the number of testing points measured in the field

\section{EXPERIMENT AND ANALYSIS}

In accordance with the above accuracy detection method, the main steps of the experiment are as follows:

(1) High resolution remote sensing select a 1:50000 standard framing in the difficult area of ortho digital image, the image from the satellite image processing, the image resolution of 1 meters; the reference data selection of topographic map data 5 on 
a 1:10000 scale; field measurement using GNSS measurement point detection.

(2) Selecting checkpoints, according to the selection requirements of inspection points, we select more than 25 characteristic points on the images as the detection points, and analyze the existing high-precision reference data, and pre select the detection points that need to be measured in the field.

(3) Field measurement, in accordance with the pre selected field measured detection point, combined with the actual situation in the field to measure the detection point.

(4) The same name points on the image and high precision reference data are measured by internal measurement, and the coordinate values of the detection points in the images to be evaluated and the high precision reference data are obtained.

(5) According to the above evaluation methods, the difference value of the coordinate, the weight of the detection point and the error in the plane position of the final sample area are calculated according to the above evaluation methods.

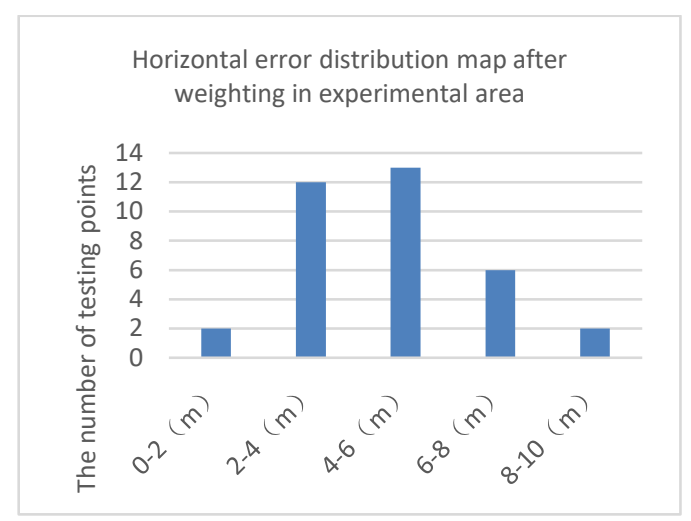

Figure 1. Horizontal error distribution map

\begin{tabular}{|l|c|}
\hline \multicolumn{1}{|c|}{ Error name } & Error value \\
\hline $\begin{array}{l}\text { The maximum horizontal error of the } \\
\text { experimental image relative to the reference } \\
\text { data }\end{array}$ & $8.35 \mathrm{~m}$ \\
\hline $\begin{array}{l}\text { The maximum horizontal error of the } \\
\text { experimental image relative to the field } \\
\text { measured data }\end{array}$ & $9.10 \mathrm{~m}$ \\
\hline $\begin{array}{l}\text { The maximum horizontal error of the reference } \\
\text { data relative to the field measured data }\end{array}$ & $5.43 \mathrm{~m}$ \\
\hline $\begin{array}{l}\text { The final horizontal error of the experimental } \\
\text { orthophoto image }\end{array}$ & $5.07 \mathrm{~m}$ \\
\hline
\end{tabular}

Table 1. Results of horizontal accuracy in experimental area

\section{CONCOLUSION}

The experimental results show that the method can not only quickly on a large area of high resolution digital orthophoto precision detection, and can guarantee the accuracy of detection accuracy, which provides a method for evaluation of high resolution remotely sensed orthophoto digital image horizontal accuracy. But for the detection of the location accuracy of Orthophoto digital images with different resolutions, the optimal proportion of all kinds of checkpoints needed to be further studied. In addition, there are many types can be used for high precision image detection data, other types of data used to test results, accuracy and efficiency, can be a variety of high precision data used in precision detection, and less use or not to use the field measurements to test the accuracy of imaging is feasible, and to further study.

\section{ACKNOWLEDGEMENTS}

This study is sponsored by National Natural Science Foundation Project of China. The project name is a study on the quality automated inspecting and testing of the land cover classification data based on deep belief neural network. The number of the project is 41671440 .

\section{REFERENCES}

Chen Zemin, Ma Ronghua, J, 2002. Precision Analysis of IKONOS Satellite Remote Sensing Image. Remote sensing technology and application, Vol.17,Part 1,pp: 46-42.

Zeng Yanwei, Tan Mingjian, etc,2009. GB/T24356-2009, Specitications for Quality Inspection and Acceptance of Surveying and Mapping Products, China National Standard Management committee, Beijing, China.

Li D R, Tong Q X, Li R X, etal,2012. Current Issues in HighResolution Earth Observation Technology. Sci China Earth Sci, Vol.55, pp:1043-1051.

Xu Dezheng, 2017 D, Research on the Evaluation Method for Image Geometric Precision of Domestic Optical Satellites, Lanzhou jiaotong University,Lanzhou,China.

Tao Wan-cheng, Zhang Yong-bin,2017A, Effect of Precision of Control Points on Geometric Correction of Remote Sensing Image, Journal of north china university of science and technology,Vol,39,Part 4,pp: 7-14.

YUAN xiuxiao, ZHANG guo, 2003A. Object Location of Satellite Imagery Under Lacking Ground Control Points. Geomatics and Information Science of Wuhan University, Vol.28, Part.5, pp:505-509.

Tang Xinming, Zhang guo, Zhu Xiaoyong, et al. 2012A. Triple Linear-array Imaging Geometry Model of Ziyuan-3 Surveying Satellite and Its Validation, Acta Geodaetica et Cartographica Sinica, Vol.41,Part2,pp:191-198.

Gong jianya. Wang Mi, Yang Bo. 2017A. High-precision geometric processing theory and method of high-resolution optical remote sensing satellite imagery without GCP, Acta Geodetica et Cartographica Sinica, Vol,46,Part10,pp:12551261.DOI:10.11947/j.AGCS.2017.20170307. 